\title{
Transanal total mesorectal excision: full steam ahead
}

\author{
S. Atallah
}

Received: 15 December 2014/ Accepted: 16 December 2014/Published online: 6 January 2015

(C) Springer-Verlag Italia Srl 2015

The article by Araujo [1] in this month's issue documents the dramatic climb of transanal total mesorectal excision (transanal TME) with the most comprehensive review on this topic to date and a revelation of published numbers that are approximately double what they were just 1 year ago [2]. While it may seem as if transanal TME materialized overnight, in actuality, its foundation had been quietly in development for three decades. The seeming sudden ascent of transanal TME is analogous to a building under construction. Developing a proper foundation is a time-intensive and critical first step. To naïve observers of a construction site, it may appear as though no progress is underway, until the foundation is completed and suddenly a building begins to rise ten stories high, filling in the skyline with striking speed.

The foundation for transanal TME began 30 years ago with the innovative efforts of Gerald Marks and later carried on with his son John Marks. In the early 1980s, G. Marks pioneered the technique of what he termed the transabdominal-transanal (TATA) operation for rectal cancers involving the distal one-third of the rectum [3], performing a meticulous dissection from below and adapting Bill Heald's concept of TME, albeit 'in reverse.' This predated the era of laparoscopy and the first TATA operations were completed with an open approach before transitioning to the use of laparoscopic assistance in the 1990s [4]. For 30 years, Marks' TATA operation laid down the foundation for modern transanal TME, spelling out very clearly why this approach was preferred for distal rectal cancer. Principally, the advantages of TATA were (a) definition of the distal margin from below, thereby

S. Atallah $(\square)$

Florida Hospital, Winter Park, FL, USA

e-mail: atallah@post.harvard.edu assuring no tumor involvement at the line of distal division and (b) obviating the need for stapling devices for transection, which do not comply with boney constraints ofin particular-the narrow male pelvis. Even when staplers could be negotiated into pelvic confines from above, there was still the risk of inadvertently stapling across tumor, thereby irreversibly compromising the oncologic integrity of the operation. While Marks' rationale behind TATA was perfectly on point, the technique required specialized retractors with limited exposure, and thus few were able to replicate this approach.

In parallel with TATA, the transanal endoscopic microsurgery (TEM) scope was developed by Buess [5] and considerable experience was gained with TEM for local excision of rectal neoplasia, though primarily for nonmalignant lesions. TEM was, for many, an unaffordable privilege of the elite; most colorectal surgeons, to this day, are not privy to TEM nor have they had formal training with this exquisite device. TEM's penetration had remained limited due to prohibitive upfront costs and also the complexity it mandates from surgeons.

During a recent lectureship webcast, a renowned colorectal surgeon remarked that, 'in [the year] 2000, TEM was dead.' This statement was not intended to discredit the otherwise precocious TEM marvel. Implicit, however, was that the technique failed to gain universal adoption by colorectal surgeons due to the reasons cited. But in the mid-2000s, an important innovation would set TATA and TEM on a collision course with one another; it would not only resurrect TEM, but it would also spawn the next generation of advanced transanal platforms. That innovation was natural orifice translumenal surgery (NOTES) [6].

Those with skepticism about NOTES asked: What for? But the innovators of the time asked: What next? It immediately set off a worldwide quest to determine what 
types of operations could be mastered with the NOTES approach. Among these leaders was Mark Whiteford, who in collaboration with NOTES authority, Lee Swanström, successfully demonstrated that transanal TME could be completed using a TEM scope and without abdominal assistance in a cadaveric model [7]. Unlike Marks, Whiteford's objective was not improved access to the distal rectum, but rather the avoidance of abdominal access trauma (the very impetus behind NOTES) [6]. This experiment was a preclinical first step to performing NOTES transanal TME in humans.

Over the years, there has been growing disenchantment with NOTES because its principle objective-to reduce abdominal access trauma-was at the expense of visceral trauma. Unfortunately, this represents a fundamental flaw of NOTES that cannot be overcome, and it is not a sound logical syllogism to assume that visceral access trauma is less significant than abdominal access trauma [8]. Notwithstanding, there are important exceptions, such as when the visceral incision is part of the planned operation. Perhaps, the most quintessential example of this NOTES approach is transanal TME, and this is an important reason why Whiteford's work could almost immediately be translated into clinical application.

That happened in 2010 when Patricia Sylla and Antonio de Lacy using a hybrid of Whiteford's technique (i.e., a TEM scope transanally with laparoscopic assistance) demonstrated feasibility of the first transanal TME in a human [9]. While this mind-over-matter surgical feat represented an important step in transitioning to the concept of the modern transanal TME, there was a lull in transanal TME's progression after this because the TEM scope (at the time, the only advanced transanal access platform) was cumbersome and the exposure proved arduous, limiting the practicality of the approach.

The modern transanal TME would require a different kind of interface. As chance would have it, in the same week (and journal) that de Lacy and Sylla published their work, our group introduced a new concept for advanced transanal surgery-a platform called transanal minimally invasive surgery (TAMIS) [10]. TAMIS was actually a tangent taken from NOTES as-for a brief whileabdominal access via the 'embryonic' umbilical orifice was considered a type of NOTES (so-called E-NOTES), and industry developed the multi-channel port likely with this concept in mind. We had gained some experience with E-NOTES, primarily for right hemicolectomy, with limited benefit. However, when we decided to place a single port transanally and use it as a platform for rectal access, we had serendipitously stumbled onto what would become one of the best applications of the multi-channel laparoscopic port-TAMIS.
Initially, we saw TAMIS as a 'poor man's TEM' and a method to perform high-quality, low-cost local excision of well-selected rectal neoplasms. But in addition, we also instinctively knew that this powerful new platform would have applications beyond local excision. This is why when we had coined the acronym, and it was kept intentionally generic as it was perceived it would have broader applications, although, candidly, at the time we did not know what those would be!

As it turns out, TAMIS would become the essential element that would perfectly meld Marks' TATA operation with Whiteford's NOTES rectal resection thereby creating a gateway to the modern transanal TME operation-that is, TAMIS-TME [11, 12]. By 2013, what started in the 1980s with TEM, TME, and TATA has laid down the critical foundation which ignited a revolution in advanced transanal surgery for colorectal surgeons worldwide [13].

Just a few years ago, it was believed that the metaphoric 'holy grail' of transanal TME was to be able to perform the operation completely via a transanal approach thereby eliminating abdominal access trauma. And while a few master surgeons have been able to demonstrate the feasibility of this approach [14-16], it is actually no longer the principle objective of transanal TME. Instead, the original objective of an 'incisionless' pure NOTES rectal operation has been usurped by a far more important realization: Improved distal rectal access allows for improved surgical management of rectal cancer, and this has the potential to (a) improve resection quality, thereby clinical outcomes and (b) decrease the incidence abdominoperineal resection (APR), thereby improving sphincter preservation rates. Thus, the prior NOTES-driven objective of avoiding abdominal access trauma (via a pure transanal NOTES approach) is not a critical requirement of the modern transanal TME. In fact, the best practice for delivering a bulky TME specimen should probably not be transanal delivery (pure NOTES) - because it could shear the envelope. Instead, the specimen should be delivered via an abdominal extraction site, and for select cases, this is a more oncologically sound approach.

The crucial reason as to why transanal TME is so valuable can be surmised with a single word-access. With the modern transanal TME (TAMIS-TME), a perfect inline vantage point is achieved. This is because the pelvis is not a 'deep hole' but rather, it takes on the configuration more similar to the capital letter ' $\mathrm{L}$,' whereby the horizontal line of ' $L$ ' represents the distal one-third of the rectum. It is this ' $\mathrm{L}$ ' configuration that (in patients positioned dorsal lithotomy) allows for direct line-of-sight to the target organ. To use a simple analogy, prior to transanal TME, it was as though we were trying to change the motor oil of an automobile from above, rather than where it is 
very easily accessed - from the undercarriage of the engine compartment.

In truth, transanal TME provides an important solution to what was heretofore the most vexing problem in rectal oncologic surgery-namely en bloc resection of tumors in the distal one-third of the rectum, especially for patients with a challenging pelvis. However, transanal TME is not without pitfalls, and it is not a panacea. There are key pearls which, as surgeons, we must collectively understand to assure the safe implementation of transanal TME and to minimize morbidity as we advance along our proficiencygain curve.

The most important pearl is that transanal TME may result in a slight, but real, increase incidence of urethral injury, especially at the level of the post-prostatic urethra and especially in the setting of anterior tumors, prior pelvic radiation (where tissue planes become adherent), and in male patients with prostatic hypertrophy or a prior history of prostatectomy. This is perhaps the single most significant morbidity that can occur that is specific to the transanal TME approach (i.e., urethral injury is extremely uncommon with low anterior resection from above, regardless of modality). In one of the largest series on transanal TME by Philippe Rouanet, the incidence of urethral injury was 2 in 30 [17], and in our own experience, we have had 1 in 45 (unpublished data). Fortunately, this transanal TME-specific complication is avoidable with appropriate patient selection and proper training with early recognition of veering off-plane during dissection.

There is a second point of caution. While transanal TME (and the $\mathrm{CO}_{2}$ dissection it generates) allows excellent visualization of Bill Heald's 'angel-hair' plane, it can also show areolar planes beyond the scope of dissection that can lead the surgeon astray. This occurs in two distinct areas: (a) at the level of the mid rectum, laterally and (b) posteriorly, at the level of the mid and upper rectum, where the rectosacral fascia that communicates with the endopelvic fascia can place the operating surgeon in a plane that is 'too deep,' thereby entering the sacral space. This does not affect TME quality and instead leads to, on occasion, encasement of the specimen in a 'double envelope,' whereby the endopelvic fascia is included in the resected specimen and from this standpoint it is not oncologically inferior. However, going off-plane can result in inadvertent injury to pelvic sidewall autonomic nerves and, posteriorly, the sacral venous plexus, thereby resulting in untoward hemorrhage. Heme-stained planes distort the surgeon's view during transanal TME and this can become a hindrance to further proceeding from below and maintaining strict and correct anatomic planes. Veering off-plane laterally can injure the autonomic pelvic plexus and nervi erigentes, as well as the sympathetic innervation from the $S 3$ root, important in function of the internal anal sphincter mechanism. Recently, Kneist et al. [18] have mapped the five key zones with potential for autonomic nerve injury during TAMIS-TME which is quite useful.

Furthermore, there are technical lessons learned that have merit and have ameliorated challenges of the current apparatus used. Take, for example, platform choice. TEM (or TEO) is an excellent platform and, for local excision of rectal neoplasms, it remains the gold standard. However, its long scope limits the ability of the surgeon to work outside of the scope axis substantially. With the existing TAMIS platform, there is an advantage of $23^{\circ}$ over the TEM scope [19], and this is a key reason why TAMIS has become the preferred platform for those performing the highest volume of this new procedure $[12,20]$. The data from the review by Araujo et al. [1] show that only a minority, 37/150 (24.7\%), of all published transanal TME cases were completed with TEM/TEO.

However, an important criticism of TAMIS is that unlike TEM, the insufflation is suboptimal and this leads to two menacing problems: (a) excessive plumes of smoke which obscure the operative field of view and (b) 'bellowing' or collapse and re-expansion of the pelvis with the cycling of $\mathrm{CO}_{2}$. Recently, a durable and simple solution has been realized-and that is use of a valveless trocar system [21]. Such a trocar is easily adaptable to the existing TAMIS platform and resolves the bellowing and excess smoke buildup due to its triple lumen system that provides independent regulation of (a) insufflation pressure, (b) $\mathrm{CO}_{2}$ delivery, and (c) smoke evacuation.

Sometimes, also, the perfect TME plane is lost due to the effects of prior radiation, whereby native planes are distorted by inflammatory changes and edema, as well as tumor-related desmoplasia. These are all to familiar posttreatment effects that leave the surgeon at a disadvantage and, just as working from above, the dissection from below can prove difficult.

Furthermore, addressing ultra-low rectal cancers brings on special challenges for transanal TME, as the lesions can extend distal to the anorectal ring and an intersphincteric dissection must then be initiated (usually using a hybrid approach). This means that the plane of dissection must begin distal to the mesorectal envelope's lower edge and therefore the dissection starts 'pre-mesorectal envelope.' Without this envelope there is no 'buffer' between tumor and the dissection plane and instead the surgeon must carefully skirt along the levator plate muscles with caution not to encroach on the nude rectal muscle tube, which could risk a positive circumferential resection margin (CRM), compromising the possibility of an R0 resection.

There are new approaches to transanal TME which are currently in development including stereotactic navigation [22] and robotic transanal surgery [23]. Robotic transanal TME could be a promising direction because of the 
potential to perform a high-quality dissection, though experience with this is limited to only three centers [2326]. Currently, the difficulty of this approach is related to negotiating the bulky robotic arms into the confined anatomic space. However, with the prospect of single-arm, multi-channel master-slave systems on the horizon, this important challenge will be overcome and the only barrier remaining will be cost-effectiveness.

Every so often, a new procedure is born that captures the attention of the surgical community and leads us in a new direction. Rarely, an entirely new category of surgery is created. In the past year, transanal TME has become a new category for rectal surgery as it is not like anything else. As such, it has become the center point of every major colorectal conference as experienced surgeons fill auditoriums to learn everything they can about it and seek specialized course training. The prediction made by Heald [27] has come to fruition and his bold statement about transanal TME 'revolutionizing the practice of rectal cancer surgery' rings true. I would add my own prediction: that over the next 5 years, transanal TME will not only become a case integrated into colorectal fellowship training curricula, but also transanal TME will become an 'index case' with a level of importance to rival the highly coveted ileoanal pouch procedure.

Despite enthusiasm, there will be non-adapters to this new kind of surgery as many will choose to await longterm data and outcomes with transanal TME, afraid of erasing 30 years of progress with rectal cancer surgery. Meanwhile, proponents argue that transanal TME is the very culmination of 30 years of progress. It is founded not by any single innovation but rather by an amalgam of seemingly different ideas and approaches applied to rectal surgery. They are: (a) TATA, (b) TEM/TAMIS, (c) TME, and (d) NOTES. Thus, transanal TME takes the most important developments in rectal cancer surgery to emerge over the last 30 years and unifies them into one operation.

As we write transanal TME into the history books with speed such that the ink has barely begun to dry, so do we begin to look toward the next chapter, still full of blank pages that draw our open pen, waiting to be filled. We are plotting a new course. Navigating our way toward the beckoning of a new frontier. Full steam ahead.

Conflict of interest Dr. Sam Atallah is a paid consultant for Applied Medical, Inc. This manuscript is the sole work and authorship of Dr. S. Atallah. This was not financially supported by any agency or person.

\section{References}

1. Araujo SE, Crawshaw B, Mendes CR, Delaney CP (2014) Transanal total mesorectal excision: a systematic review of the experimental and clinical evidence. Tech Coloproctol. doi:10. 1007/s10151-014-1233-x

2. Emhoff IA, Lee GC, Sylla P (2014) Transanal colorectal resection using natural orifice translumenal endoscopic surgery (NOTES). Dig Endosc 26(Suppl 1):29-42

3. Marks G, Mohiuddin M, Rakinic J (1991) New hope and promise for sphincter preservation in the management of cancer of the rectum. Semin Oncol 18:388-398

4. Marks J, Nassif G, Schoonyoung H et al (2013) Sphincter-sparing surgery for adenocarcinoma of the distal $3 \mathrm{~cm}$ of the true rectum: results after neoadjuvant therapy and minimally invasive radical surgery or local excision. Surg Endosc 27:4469-4477

5. Buess G, Theiss R, Günther M, Hutterer F, Pichlmaier H (1985) Endoscopic surgery in the rectum. Endoscopy 17:31-35

6. Rattner D, Kalloo A, ASGE/SAGES Working Group (2006) ASGE/SAGES working group on natural orifice translumenal endoscopic surgery. Surg Endosc 20:329-333

7. Whiteford MH, Denk PM, Swanström LL (2007) Feasibility of radical sigmoid colectomy performed as natural orifice translumenal endoscopic surgery (NOTES) using transanal endoscopic microsurgery. Surg Endosc 21:1870-1874

8. Atallah S, Martin-Perez B, Keller D, Burke J, Hunter L (2015) Natural-orifice transluminal endoscopic surgery. Br J Surg. doi:10.1002/bjs. 9710

9. Sylla P, Rattner DW, Delgado S, Lacy AM (2010) NOTES transanal rectal cancer resection using transanal endoscopic microsurgery and laparoscopic assistance. Surg Endosc 24:1205-1210

10. Atallah S, Albert M, Larach S (2010) Transanal minimally invasive surgery: a giant leap forward. Surg Endosc 24:2200-2205

11. Atallah S, Albert M, DeBeche-Adams T, Nassif G, Polavarapu H, Larach S (2013) Transanal minimally invasive surgery for total mesorectal excision (TAMIS-TME): a stepwise description of the surgical technique with video demonstration. Tech Coloproctol 17:321-325

12. Atallah S, Martin-Perez B, Albert M et al (2014) Transanal minimally invasive surgery for total mesorectal excision (TAMIS-TME): results and experience with the first 20 patients undergoing curative-intent rectal cancer surgery at a single institution. Tech Coloproctol 18:473-480

13. Lirici MM, Kanehira E, Melzer A, Schurr MO (2014) The outburst age: how TEM ignited the MIS revolution. Minim Invasive Ther Allied Technol 23:1-4

14. Chouillard E, Chahine E, Khoury G et al (2014) NOTES total mesorectal excision (TME) for patients with rectal neoplasia: a preliminary experience. Surg Endosc 28:3150-3157

15. Zhang H, Zhang YS, Jin XW, Li MZ, Fan JS, Yang ZH (2013) Transanal single-port laparoscopic total mesorectal excision in the treatment of rectal cancer. Tech Coloproctol 17:117-123

16. Leroy J, Barry BD, Melani A, Mutter D, Marescaux J (2013) Noscar transanal total mesorectal excision: the last step to pure NOTES for colorectal surgery. JAMA Surg 148:226-230 discussion 231

17. Rouanet P, Mourregot A, Azar CC et al (2013) Transanal endoscopic proctectomy: an innovative procedure for difficult resection of rectal tumors in men with narrow pelvis. Dis Colon Rectum 56:408-415

18. Kneist W, Rink AD, Kauff DW, Konerding MA, Lang H (2014) Topography of the extrinsic internal anal sphincter nerve supply during laparoscopic-assisted TAMIS TME: five key zones of risk from the surgeons' view. Int J Colorectal Dis. doi:10.1007/ s00384-014-2026-4

19. Martin-Perez B, Andrade-Ribeiro GD, Hunter L, Atallah S (2014) A systematic review of transanal minimally invasive surgery (TAMIS) from 2010 to 2013. Tech Coloproctol 18:775-788 
20. Fernández-Hevia M, Delgado S, Castells A et al (2014) Transanal total mesorectal excision in rectal cancer: short-term outcomes in comparison with laparoscopic surgery. Ann Surg. doi:10.1097/ SLA.0000000000000865

21. Bislenghi $\mathrm{G}$, Wolthuis AM, Overstraeten ADB, D'Hoore A (2014) AirSeal system insufflator to maintain a stable pneumorectum during TAMIS. Tech Coloproctol. doi:10.1007/s10151014-1244-7

22. Atallah S, Nassif G, Larach S (2014) Stereotactic navigation for TAMIS-TME: opening the gateway to frameless, image-guided abdominal and pelvic surgery. Surg Endosc. doi:10.1007/s00464014-3655-y

23. Atallah S, Nassif G, Polavarapu H et al (2013) Robotic-assisted transanal surgery for total mesorectal excision (RATS-TME): a description of a novel surgical approach with video demonstration. Tech Coloproctol 17:441-447

24. Atallah S, Martin-Perez B, Pinan J et al (2014) Robotic transanal total mesorectal excision: a pilot study. Tech Coloproctol 18:1047-1053

25. Gómez Ruiz M, Parra IM, Palazuelos CM et al (2015) Roboticassisted laparoscopic transanal total mesorectal excision for rectal cancer: a prospective pilot study. Dis Colon Rectum 58:145-153

26. Verheijen PM, Consten EC, Broeders IA (2014) Robotic transanal total mesorectal excision for rectal cancer: experience with a first case. Int J Med Robot. doi:10.1002/rcs.1594

27. Heald RJ (2013) A new solution to some old problems: transanal TME. Tech Coloproctol 17:257-258 